\title{
Generadores Síncronos de Alta Velocidad. Una Evaluación Crítica
}

\author{
Fernando Martínez ${ }^{(1)}$ y Pere Andrada(2) \\ (1) Escuela de Ingeniería Minera e Industrial, Depto. de Ingeniería Eléctrica, Electrónica, Automática y \\ Comunicaciones, Universidad de Castilla La Mancha, Plaza Manuel Meca 1, 13400 Almadén, (Ciudad \\ Real), España. (e-mail: fernando.mgarcia@uclm.es) \\ (2) Escola Politècnica Superior d'Enginyeria, Depto. de Ingeniería Eléctrica, Universidad Politécnica de \\ Cataluña, Victor Balaguer 1, 08800 Vilanova i La Geltrú, (Barcelona), España. \\ (e-mail: pere.andrada@upc.edu)
}

Recibido Ago. 29, 2016; Aceptado Nov. 2, 2016; Versión final Dic. 7, 2016, Publicado Abr. 2017

\begin{abstract}
Resumen
Considerando que existen restricciones electromagnéticas, mecánicas y térmicas que condicionan el diseño de las máquinas eléctricas de alta velocidad, se establecen los criterios que conducen a una adecuada selección de la máquina. Además, teniendo en cuenta el estado actual de la tecnología, se presentan los valores críticos en la relación (potencia de salida)/(velocidad de rotación). El artículo se centra en los generadores síncronos de alta velocidad, especialmente en los generadores con imanes permanentes, los alternadores inductores y los alternadores con conmutación de flujo, haciendo hincapié en sus fundamentos constructivos y en sus ventajas e inconvenientes. El análisis se completa con una evaluación crítica que los clasifica, de acuerdo con unos requisitos previamente definidos. Aunque la decisión de la elección final está muy condicionada por la aplicación de interés, puede afirmarse que, en general, los generadores síncronos con imanes permanentes en el rotor constituyen la mejor opción.
\end{abstract}

Palabras clave: máquinas eléctricas de alta velocidad; generadores síncronos; alta frecuencia; alternadores inductores

\section{High-Speed Synchronous Generators. A Critical Assessment}

\begin{abstract}
Considering that there are electromagnetic, mechanical and thermal constraints that determine the design of high-speed electrical machines, the criteria that lead to adequate selection of the machine are established in this work. Moreover, taking into account the current state of technology, the critical values in the ratio (output power)/(rotation speed), are presented. The paper focuses on the high-speed synchronous generators, especially in permanent magnet generators, inductor alternators and flux switching alternators, emphasizing their constructive fundamentals and their advantages and disadvantages. The analysis is completed with a critical assessment that classifies them according to previously defined requirements. Although the decision of the final choice is largely determined by the application of interest, it can be said that, in general, synchronous generators with permanent magnets in the rotor are the best option.
\end{abstract}

Keywords: high-speed electrical machines; synchronous generators; high-frequency; inductor alternators 


\section{INTRODUCCIÓN}

Actualmente existe un gran interés en la utilización de máquinas eléctricas de alta velocidad de rotación, ya que, al posibilitar la eliminación de la transmisión mecánica, presentan ventajas tales como mayor densidad de potencia, mayor rigidez mecánica, menor ruido, reducción del volumen y del peso del conjunto, menor desgaste y menores costes de mantenimiento. En los últimos años se han realizado importantes aportaciones al estudio de las máquinas de alta velocidad de rotación, en aplicaciones industriales para accionamientos de turbocompresores (Zhang et al., 2016), bombas centrífugas y de vacío (Huang y Fang, 2016), y máquinas herramientas (Gieras, 2014). También han tenido un importante impacto en el ámbito de la generación eléctrica, especialmente en la generación distribuida con microturbinas (Zwissig et al., 2009), (Figura 1), en alternadores para aeronaves (Lahne et al., 2016), en volantes de inercia para almacenamiento de energía (Kumar et al., 2016), en el lanzamiento y guiado de misiles (McNab, 2015) y en armamento basado en energía electromagnética dirigida (Gieras, 2012).

Estas aplicaciones abarcan un rango de potencias entre $0,1 \mathrm{~kW}$ hasta $10 \mathrm{MW}$, con velocidades de rotación comprendidas entre 10 mil y 500 mil rpm. Hay que tener en cuenta que el concepto de alta velocidad está ligado a un elevado valor de la velocidad periférica o tangencial del rotor, $v,(100-300 \mathrm{~m} / \mathrm{s})$, y no necesariamente a una elevada velocidad de rotación, $n$, lo que lleva implícito tener presente el tamaño de la máquina. Aunque diversos tipos de generadores eléctricos rotativos han sido utilizados en el ámbito de las máquinas eléctricas de alta velocidad (Bartolo et al., 2013), este artículo se centra en los generadores síncronos, excluyendo otros tipos de generadores, como los asíncronos y los de reluctancia autoconmutados.

El objetivo del artículo es hacer una evaluación crítica de los generadores síncronos de alta velocidad, incluyendo a los alternadores inductores, (inductor alternators), y a los alternadores con conmutación de flujo, (flux switching alternators), proporcionando una guía para su adecuado diseño y selección. El artículo se ha organizado presentando, en primer lugar, las limitaciones de las máquinas eléctricas de alta velocidad, para seguidamente describir los distintos tipos de generadores síncronos, continuar con una evaluación crítica de los generadores síncronos de alta velocidad y finalizar con las conclusiones derivadas de este estudio.

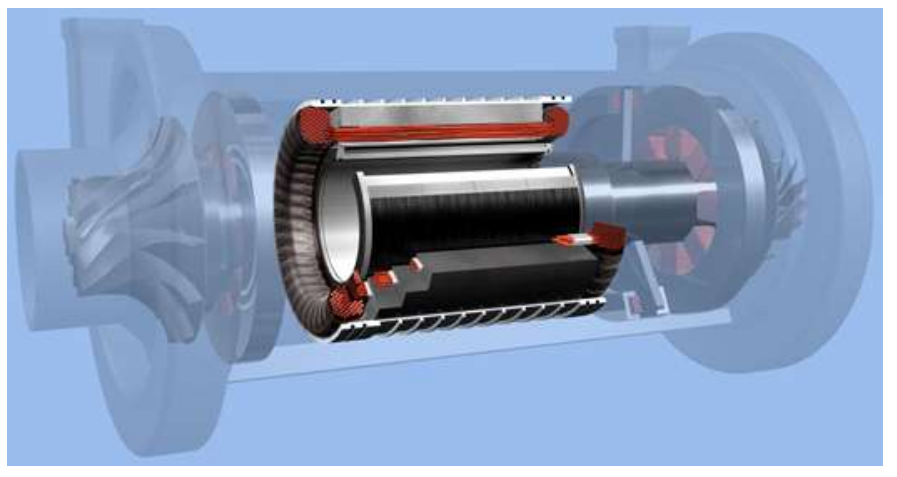

Fig. 1: Generador síncrono para microturbina, de 200 kW y 21.000 rpm.

Cortesía de e + k Elektromaschinen und Antriebe (Switzerland).

\section{LIMITACIONES DE LAS MÁQUINAS ELÉCTRICAS DE ALTA VELOCIDAD}

Las máquinas eléctricas de alta velocidad presentan limitaciones mecánicas y térmicas, además de restricciones impuestas por el convertidor electrónico de potencia asociado, que condicionan su diseño.

\section{Pérdidas}

Las pérdidas en las máquinas eléctricas se clasifican en magnéticas, eléctricas y mecánicas, y todas ellas aumentan considerablemente cuando lo hace la velocidad de rotación. En las máquinas síncronas existe una relación entre la frecuencia, $f$, la velocidad de rotación, $n(\mathrm{rpm})$ y el número de pares de polos, $p$, dada por la expresión: $f=(p \cdot n) / 60$. Las pérdidas en el cobre, (eléctricas), son debidas al calentamiento de los conductores por efecto Joule. Se generan, además, pérdidas adicionales como consecuencia de los efectos pelicular y de proximidad. Estas pérdidas varían con $f^{2}$, lo que obliga a utilizar conductores trenzados 0 conductores tipo Litz en las aplicaciones de alta velocidad de rotación (Reddy et al., 2009). Las pérdidas en el hierro, (magnéticas), en su conjunto, considerando las pérdidas por histéresis, por corrientes parásitas y las adicionales, varían aproximadamente con el producto del cuadrado de la inducción magnética y el cuadrado de la frecuencia, ( $B^{2} . f^{2}$ ) (Huynh et al., 2009). Esta circunstancia obliga a utilizar, en las aplicaciones de alta velocidad de rotación, chapa magnética de muy bajas pérdidas específicas en el hierro y de pequeño espesor o materiales magnéticos especiales (Fernando et al., 2016). 
Las pérdidas mecánicas son debidas a la fricción con el aire y al rozamiento de los rodamientos. Varían proporcionalmente a $n^{y}$, (con y $=2-3$, dependiendo de la condición superficial del rotor y del estator) (Binder y Canders, 2014). Como consecuencia del notable incremento de las pérdidas con la velocidad de rotación, especialmente las pérdidas en el hierro y las pérdidas por fricción con el aire, los efectos térmicos derivados de éstas, fundamentalmente el incremento de temperatura y sus consecuencias en los aislamientos, en la resistencia de los devanados y en los imanes permanentes, hacen que el sistema de refrigeración sea uno de los aspectos que requiere mayor atención en el diseño de una máquina eléctrica de alta velocidad (Tüisüz et al., 2015).

\section{Materiales y consideraciones dimensionales}

La operación a alta velocidad introduce enormes esfuerzos mecánicos en el rotor debido a la fuerza centrífuga, que genera la componente tangencial de fuerza de tracción en el material. El esfuerzo es proporcional a la densidad del material y al cuadrado de la velocidad tangencial, $\left(\sigma_{t} \sim \rho v^{2}\right)$. Así pues, el límite elástico del material del rotor, la máxima velocidad tangencial y el valor máximo de la velocidad de rotación determinan el diámetro de la máquina (Tenconi et al., 2014). Estas circunstancias condicionan las dimensiones del rotor y la elección del material con el que éste se ha de construir, lo que no es tarea fácil ya que los materiales más adecuados, aquellos con elevado límite elástico y baja densidad, no son materiales magnéticos. En el caso de que en el rotor haya devanados o imanes permanentes, deben utilizarse retenedores de fibra de carbono, para garantizar su fijación (Binder et al., 2006). El diseño del rotor debe hacerse considerando, además, las frecuencias naturales de resonancia, para evitar vibraciones (Ede et al., 2002).

\section{Rodamientos}

En este tipo de máquinas eléctricas es necesario prestar especial atención a los rodamientos, que han de funcionar, de forma estable y durante un largo periodo de tiempo, a elevadas velocidades, deben hacer frente a las perdidas por fricción, soportar la rigidez torsional y axial del eje y han de adaptarse a las cargas mecánicas solicitadas. En las máquinas eléctricas de alta velocidad se utilizan diferentes tipos de rodamientos: mecánicos, de aire (o de fluido líquido) y magnéticos (Borisavljevic, 2011). Para las velocidades de rotación más elevadas, los rodamientos magnéticos o de aire constituyen la mejor elección. En la Tabla 1 se indican las ventajas e inconvenientes de los diferentes tipos de rodamientos utilizados en las máquinas eléctricas de alta velocidad.

Tabla 1: Ventajas e inconvenientes de los diferentes tipos de rodamientos.

\begin{tabular}{|l|l|l|}
\hline \multicolumn{1}{|c|}{ Tipo } & \multicolumn{1}{|c|}{ Ventajas } & \multicolumn{1}{c|}{ Inconvenientes } \\
\hline $\begin{array}{l}\text { Mecánicos } \\
\text { (Bolas metálicas o cerámicas) }\end{array}$ & $\begin{array}{l}\text { Robustez } \\
\text { Bajo coste }\end{array}$ & $\begin{array}{l}\text { Desgaste. Necesidad de } \\
\text { lubricación y de mantenimiento. } \\
\text { Temperatura limitada }\end{array}$ \\
\hline $\begin{array}{l}\text { Aire o fluido } \\
\text { (Película a presión entre el } \\
\text { rodamiento y el alojamiento) }\end{array}$ & $\begin{array}{l}\text { Sin fricción ni degaste } \\
\text { Gran precisión } \\
\text { Alta rigidez } \\
\text { Bajo mantenimiento }\end{array}$ & $\begin{array}{l}\text { Inestabilidad. Baja capacidad de } \\
\text { carga. Requerimientos de } \\
\text { geometría perfecta. Necesidad de } \\
\text { precarga. Susceptibilidad a la } \\
\text { suciedad y a la temperatura }\end{array}$ \\
\hline $\begin{array}{l}\text { Magnéticos } \\
\text { (Electroimanes que hacen } \\
\text { levitar el rotor) }\end{array}$ & $\begin{array}{l}\text { Sin fricción ni desgaste. Posicionamiento } \\
\text { durante la rotación. Fuerza y amortiguamiento } \\
\text { ajustables. Diseño modular. Operación en } \\
\text { condiciones muy adversas. Fácil seguimiento } \\
\text { mediante monitorización. Sin mantenimiento }\end{array}$ & $\begin{array}{l}\text { Complejidad. Necesidad de } \\
\text { control. Baja fiabilidad. Necesidad } \\
\text { de fuente de alimentación, } \\
\text { sensores y electrónica auxiliar. } \\
\text { Alto precio }\end{array}$ \\
\hline
\end{tabular}

\section{Convertidor electrónico de potencia}

Las máquinas eléctricas de alta velocidad, ya sea funcionando como motor o como generador, tienen asociado un convertidor electrónico de potencia, generalmente un inversor, que en el caso del motor permite la regulación de la velocidad del accionamiento y, en el caso del generador, la regulación de la tensión y la frecuencia de salida. La alta frecuencia limita la potencia y el control en los inversores (Li et al., 2016). La frecuencia de conmutación del inversor tiene que ser bastante mayor que la frecuencia fundamental de la tensión de salida del generador, para limitar los armónicos. Si hay un alto coeficiente de distorsión armónico en el inversor, se inducirán pérdidas adicionales en el generador. Cuando la frecuencia fundamental de la tensión del generador es muy alta, la modulación por ancho de pulso, (PWM), puede no ser adecuada (Moghaddam, 2014). Las estrategias de control y sus algoritmos también requieren de un mayor ancho de banda a altas frecuencias. 


\section{Consideraciones de diseño}

Para optimizar el diseño de las máquinas eléctricas de corriente alterna, resulta conveniente partir de la expresión de la potencia aparente en el entrehierro, $S_{\delta}(V A)$, indicada en (1). (Binder y Canders, 2014). Esta ecuación expresa que la potencia de salida de la máquina es función de su tamaño, de las variables electromagnéticas y de la velocidad de rotación.

$S_{\delta}=\left(\frac{\pi^{2} \sqrt{2}}{120}\right) \cdot k_{W} \cdot A \cdot B \cdot D^{2} \cdot L \cdot n$

Con:

$k_{w}$ : Factor de devanado $(\sim 0,9-0,95)$

$A$ : Carga eléctrica específica (producto de la corriente por el número de conductores, por unidad de longitud de la periferia del entrehierro, $\mathrm{A} / \mathrm{m}$ )

$B$ : Carga magnética específica (densidad de flujo en el entrehierro, $\mathrm{T}$ )

$D$ : Diámetro del rotor $(\mathrm{m})$

$L$ : Longitud axial de la parte magnéticamente activa $(\mathrm{m})$

$n$ : Velocidad de rotación (rpm)

Al aumentar la velocidad de rotación disminuyen las dimensiones de la máquina como consecuencia de los esfuerzos mecánicos; igualmente lo hacen la inducción magnética en el entrehierro, (B), y la carga eléctrica específica, (A), para limitar el calentamiento y disminuir así las pérdidas. Por lo tanto, la potencia de salida de la máquina disminuye. Esta circunstancia ha sido cuantificada por diversos autores, que han establecido correlaciones potencia-velocidad de rotación a partir de los datos de máquinas construidas que pueden encontrarse en la literatura técnica.

Así Moghaddam (2014) ha propuesto el denominado índice HS, (high speed), que es igual al producto de la potencia nominal, (MW), por la velocidad nominal de rotación, ( $\mathrm{krpm})$. El valor del índice HS resulta significativo respecto a las limitaciones en la potencia de salida como consecuencia de la alta velocidad de rotación. Para $\mathrm{n}<20.000 \mathrm{rpm}$, los esfuerzos mecánicos en el rotor son la principal causa limitadora, por lo que el factor determinante es la velocidad periférica del rotor. El índice HS es independiente de $n$ y solo depende de la velocidad periférica al cuadrado. Se obtienen, actualmente, valores máximos de HS: 180 200 , dependiendo de los materiales utilizados. Para $n>20.000$ rpm, además de los esfuerzos mecánicos en el rotor, las pérdidas adicionales limitan la máxima capacidad de potencia, debido a las limitaciones térmicas. El índice HS es proporcional a $v_{\max }{ }^{3} / n^{2}$, por lo que disminuye muy rápidamente al aumentar $n$. Se alcanzan actualmente valores HS 20 a 50.000 rpm, 10 a 100.000 rpm, e inferiores a 2 a velocidades superiores.

Gerada et al. (2014) utilizan como índice indicativo, índice G, el producto de la velocidad por la raíz cuadrada de la potencia, $\mathrm{G}=n \cdot \sqrt{P}$, $(\mathrm{rpm} \times \mathrm{kW})$, lo que significa suponer: $P=K / n^{2}$. Los problemas dinámicos son mínimos cuando este producto es inferior a $10^{5}$, son importantes cuando el índice está comprendido entre $5 \cdot 10^{5}$ y $10^{6}$ y son muy agudos cuando se supera el valor $10^{6}$. Valores superiores a $10^{6}$ implican velocidades periféricas muy elevadas y solo se han alcanzado hasta ahora en casos excepcionales. Considerando criterios de diseño optimizados, Zheng (2014) obtiene para los generadores síncronos de alta velocidad valores de $\mathrm{K} \sim 4.10^{11}-10^{12}$, dependiendo de la configuración de la máquina.

Otros autores han propuesto, en distintas épocas, correlaciones entre la potencia y la velocidad. Cabe destacar las propuestas por Maeda et al. (1995), $\left(P=6,2 \cdot 10^{6} / n^{3,3}, P=k W, n=r p m / 1000\right)$ y por Binder y Schneider (2007), ( $\log n=4,27-0,275 \log P, P=W, n=r p s)$. Como consecuencia de las sucesivas mejoras en la tecnología, los prototipos construidos más recientemente se sitúan por encima de estas líneas de correlación.

En la Figura 2 se recogen datos publicados de prototipos de máquinas eléctricas, de diferentes tipos, construidos durante los últimos años, en generadores y motores, así como las correlaciones de Maeda, (MA), y Binder, $(\mathrm{BI})$, y los índices de Gerada $\left(G=5 \cdot 10^{5}\right.$ y $\left.G=10^{6}\right)$. En muchas referencias utilizadas en el artículo se hace alusión a combinaciones de valores nominales: potencia - velocidad de rotación, de máquinas eléctricas que han sido construidas. Se han seleccionado los valores más críticos, para ser representados en la Figura 2. Cabe citar, como referencias en las que pueden encontrarse una mayor cantidad de ejemplos: Antipov y Danilevich (2007); Bartolo et al. (2013); Binder y Canders (2014); Gerada et al. (2014); Moghaddam (2014); Rahman et al. (2004); Tenconi et al. (2014) y Zwyssig et al. (2009). 


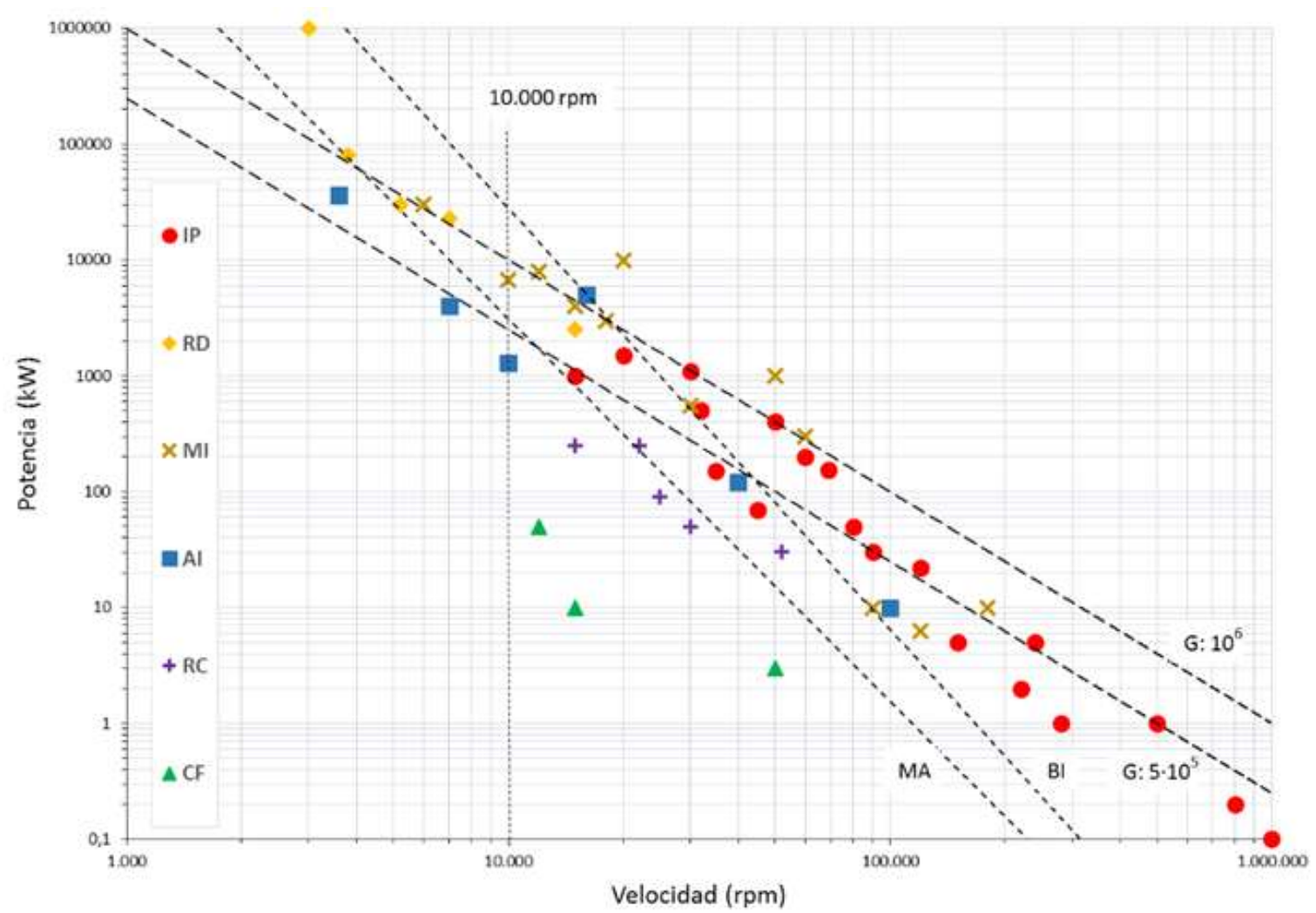

Fig. 2: Limitación de la potencia en función de la velocidad de rotación. (IP: Rotor con imanes permanentes, RD: Rotor devanado, MI: Motor de inducción, Al: Alternador inductor homopolar, RC: Máquina de reluctancia conmutada, CF: Alternador con conmutación de flujo).

Todas las limitaciones consideradas constituyen retos tecnológicos que han de tenerse en cuenta en el diseño de las máquinas eléctricas de alta velocidad de rotación y, en particular, en el caso de los generadores síncronos. Para hacer frente a estas limitaciones deberían tenerse en cuenta las consideraciones de diseño resumidas en la Tabla 2, que han sido recopiladas por los autores tras el análisis de las referencias consultadas.

Tabla 2: Consideraciones de diseño para los generadores síncronos de alta velocidad.

\begin{tabular}{|l|l|}
\hline Mecánicas & $\begin{array}{l}\text { Utilizar rodamientos adecuados para la alta velocidad. Simplicidad y robustez del rotor. } \\
\text { Limitación de las dimensiones del rotor por la velocidad tangencial y por las propiedades de los } \\
\text { materiales utilizados. Óptima sujeción de los imanes del rotor cuando sean utilizados. Alta } \\
\text { velocidad crítica del eje }\end{array}$ \\
\hline Electromagnéticas & $\begin{array}{l}\text { Minimizar las pérdidas magnéticas utilizando laminaciones muy delgadas o materiales } \\
\text { especiales. Minimizar las pérdidas eléctricas utilizando hilos trenzados o conductores Litz wire. } \\
\text { Utilizar imanes permanentes de alta energía, alta temperatura y resistentes a la } \\
\text { desmagnetización. Optimizar el diseño de dientes y ranuras en estator y rotor. Minimizar } \\
\text { armónicos de tensión y corriente para disminuir las pérdidas por deslizamientos de los campos } \\
\text { magnéticos }\end{array}$ \\
\hline Térmicas & $\begin{array}{l}\text { Usar aislamientos eléctricos de la mayor clase térmica posible. Buscar soluciones para la } \\
\text { evacuación de calor en el rotor. Diseñar un sistema de refrigeración sencillo y eficaz }\end{array}$ \\
\hline Electrónica de & $\begin{array}{l}\text { Adecuación de controladores y sensores. Optimizar los circuitos de la electrónica de potencia } \\
\text { para la conmutación a altas frecuencias }\end{array}$ \\
\hline Otras & $\begin{array}{l}\text { Diseño simple y compacto. Fiabilidad. Alto rendimiento. Reversibilidad para funcionar como } \\
\text { motor. Bajo coste de producción de la máquina y de la electrónica de potencia. Bajo } \\
\text { mantenimiento }\end{array}$ \\
\hline
\end{tabular}

\section{DIFERENTES TIPOS DE GENERADORES SÍNCRONOS}

En esta sección se presenta la descripción física, el principio fundamental de funcionamiento y las ventajas e inconvenientes, en el funcionamiento a alta velocidad de rotación, de los diferentes tipos de alternadores síncronos de accionamiento directo: alternadores síncronos convencionales, con el rotor devanado (ASRD) o con imanes permanentes (ASIP), alternadores inductores (Al) y alternadores con conmutación de flujo $(\mathrm{ACF})$. 


\section{El Alternador Síncrono Convencional}

El ASRD tiene un núcleo ranurado en el estator, laminado, que alberga un devanado trifásico distribuido, y un rotor con un devanado de excitación, alimentado por corriente continua. La corriente llega al rotor a través de contactos formados por dos anillos y escobillas o utilizando diodos rotativos. Los ASRD de flujo radial funcionan por debajo de $10.000 \mathrm{rpm}$ y presentan dos grandes problemas a alta velocidad de rotación: el equilibrado de las partes móviles y las pérdidas eléctricas. El uso de imanes permanentes elimina las bobinas de excitación y los contactos deslizantes, lo que aumenta la eficiencia. La disposición de los imanes, ya sea en la superficie del rotor y en contacto con el entrehierro, (imanes exteriores), o ubicados dentro del rotor y asociados con piezas polares, (imanes interiores), condiciona el comportamiento magnético de la máquina (Arumugan et al., 2014).

Los imanes utilizados en los alternadores síncronos de alta velocidad se configuran empleando tierras raras, y tienen una inducción remanente, un campo coercitivo y una energía específica muy elevados. Estos imanes están compuestos de neodimio, hierro y boro, con propiedades magnéticas que disminuyen al aumentar la temperatura y son sensibles a la corrosión, por lo que se les debe dar un recubrimiento metálico (Kim et al., 2016). También se utilizan imanes de samario y cobalto, mucho más caros pero cuyas propiedades magnéticas varían muy poco con la temperatura, o materiales superconductores cerámicos, constituidos por itrio, bario y óxido de cobre (Leão, 2010). El inducido en los ASIP puede ser convencional, con un devanado distribuido en ranuras, Figura 3.a, o sin ranuras, Figura 3.b (Ganev, 2014). La ausencia de ranuras reduce el efecto de las corrientes parásitas en el hierro del estator, debidas a la variación del flujo magnético. El valor de estas corrientes inducidas aumenta con la velocidad relativa entre el campo magnético giratorio y el estator, siendo necesario disipar el calor generado.

En los ASIP de alta velocidad, las configuraciones bipolares son una exigencia y el inducido sin ranuras, (o con un reducido número de ranuras), y con imanes exteriores es la mejor solución (Uzhegov et al., 2016). La principal ventaja de los ASIP es su elevada densidad de potencia, sin embargo, el problema fundamental es la fijación de los imanes al rotor, que hace necesario disponer de un manguito envolvente para la retención de los imanes, generalmente de fibra de carbono (Zhang et al., 2015). En algunos casos el ASIP puede ser de flujo axial o de rotor de disco, con los imanes montados en el disco rotórico (Wrobel et al., 2015).

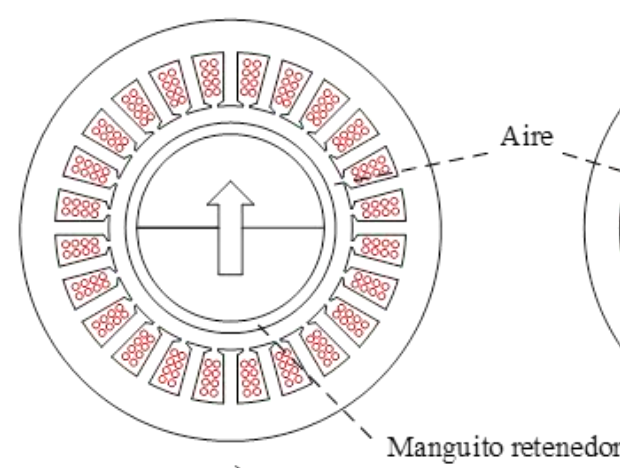

a)

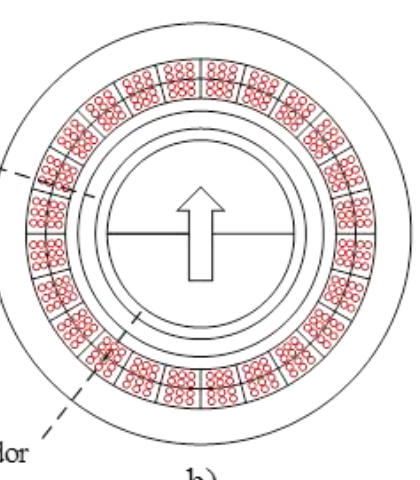

b)

Fig. 3: Sección transversal de generadores síncronos de imanes permanentes:

a) Con inducido ranurado; b) Con inducido sin ranuras.

\section{El Alternador Inductor}

El Al es un tipo de generador síncrono en el que los devanados de excitación y del inducido están en el estator y se configura el rotor mediante un núcleo dentado, sin bobinados, imanes permanentes o jaulas. La generación se produce cuando el devanado de excitación se alimenta mediante corriente continua y la variación en la reluctancia, causada por la rotación del rotor, induce una tensión alterna en el devanado del inducido. La frecuencia de la tensión inducida viene dada por $f=Z_{r} \cdot n / 60$, siendo $Z_{r}$ el número de dientes del rotor y $n$ la velocidad de rotación, en rpm.

Hay dos tipos de alternadores inductores, el homopolar (AlHO) y el heteropolar (AlHE). En los AlHO los flujos ligados a todas las bobinas del inducido tienen el mismo sentido. Este tipo de máquina, mostrado esquemáticamente en la Figura 4, consta de dos núcleos laminados en el estator, sujetos por un bastidor común. Estos núcleos están ranurados para alojar los devanados de alta frecuencia y pueden ser lisos o dentados. El devanado de excitación consiste en una única bobina central, fija al estator y montada en la periferia interior del bastidor, que produce el flujo homopolar indicado por la línea de puntos. El rotor consta de dos núcleos laminados dispuestos sobre una cruceta maciza. Estos núcleos están dentados con ranuras 
abiertas y el número de dientes puede ser igual o diferente al de los núcleos del estator. Los dientes de un núcleo se desplazan, con respecto a los dientes del otro núcleo, la mitad del paso de diente. Debido a esto, la magnitud del flujo total ligado a la bobina de excitación no varía y no se induce en ella ninguna f.e.m. alterna (Ye et al., 2015).

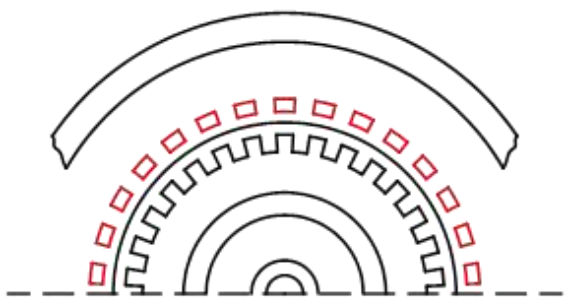

a)

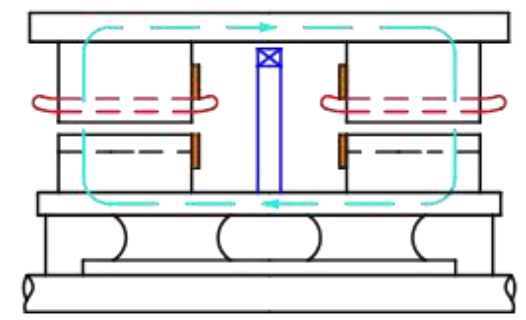

b)

Fig. 4: Alternador Inductor Homopolar: a) Sección frontal; b) Vista lateral.

Las configuraciones heteropolares, con un único rotor, se desarrollaron con posterioridad a las homopolares. En 1901 G. Guy, (Francia), patentó un AlHE con el estator dentado (Davies e Higginson, 1975), Figura 5.a, y en 1914 K. Schmidt, (Alemania), patentó un AlHE con el estator liso denominado tipo Lorentz (Davies y Lay, 1968), Figura 5.b. Posteriormente se han ido introduciendo modificaciones, en las configuraciones iniciales de los AlHE de los tipos Guy y Lorentz, que han proporcionado mejores prestaciones (Walker, 1942).

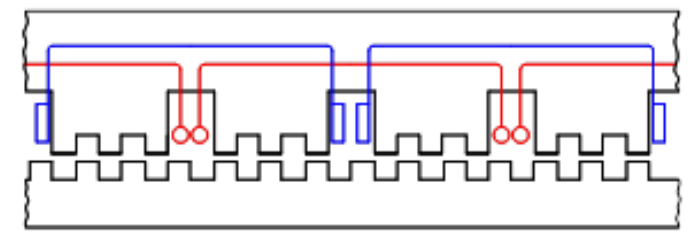

a)

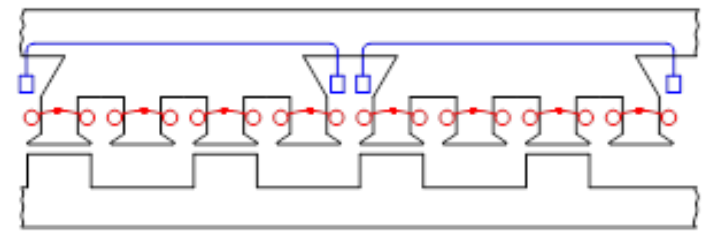

b)

Fig. 5: Alternador Inductor Heteropolar: a) Tipo Guy; b) Tipo Lorentz.

Los Al se desarrollaron desde finales del siglo XIX, fundamentalmente formando parte de convertidores electromecánicos, para la generación de energía eléctrica en alta frecuencia. A lo largo del siglo XX su uso se limitó a aplicaciones tales como la fusión eléctrica, soldadura eléctrica y tratamientos térmicos que requerían fuentes de alimentación con frecuencias comprendidas entre 1.000 y $10.000 \mathrm{~Hz}$ (Walker, 1946). Actualmente hay un renovado interés por los Al, que se están utilizando en los sistemas de iluminación y aire acondicionado de los ferrocarriles (Pillai et al., 2008), en grandes vehículos especiales para minería y obra civil (Milspec Manufacturing) y, especialmente, en aplicaciones a altas velocidades de rotación, acoplados a un volante de inercia (Severson et al., 2015) o al eje de una turbina de gas, en generadores superconductores (Sivasubramaniam et al., 2009).

Las ventajas de la utilización del Al radican, fundamentalmente, en tener un rotor sin devanados, por lo que no son necesarios anillos ni escobillas, y carecer de imanes permanentes. Puede ser utilizado hasta con una anchura de diente de rotor de $1 \mathrm{~mm}$, y girar a velocidades superiores a $200 \mathrm{mil}$ rpm, ya que la velocidad periférica máxima está limitada únicamente por las propiedades mecánicas del material del rotor. El coste de fabricación es bajo y, prácticamente, no necesita mantenimiento. El principal inconveniente del Al es que, a igualdad de potencia de salida, requiere mayores dimensiones, y por lo tanto mayor masa, que un ASIP, debido a que el flujo en el inducido varía entre los valores máximo y mínimo, pero no cambia de sentido. Además, la frecuencia de salida en el AI, para una misma velocidad de rotación, es mayor que en el ASIP.

\section{El Alternador con Conmutación de Flujo}

El ACF puede considerarse una evolución del AIHE y fue dado a conocer por Rauch y Johnson (1955). Se compone, en el caso más sencillo, de un estator laminado con cuatro polos, en el que el devanado de excitación y los devanados del inducido están dispuestos alternativamente. El devanado de excitación puede ser sustituido por imanes permanentes, como se muestra en la Figura 6.a. El rotor está formado por laminaciones, con dos polos salientes. A medida que el rotor gira, el flujo magnético sigue unas trayectorias que contienen al devanado de excitación, al inducido y al rotor. Cuando el rotor ha girado $180^{\circ}$ el flujo ligado a las bobinas del inducido ha invertido su dirección, pero mantiene el mismo valor. El ACF tiene la misma simplicidad del rotor que el Al pero añade la ventaja de que la conmutación del flujo en cada una de las bobinas del inducido proporciona una mayor variación en el valor de la densidad de flujo magnético. 
Las diferentes topologías actuales están basadas en criterios que tienen en cuenta el tipo de excitación, (corriente continua, imanes permanentes, híbrida), la orientación del flujo en el entrehierro, (radial o axial) y la construcción del núcleo del estator y del rotor (forma y número de dientes) (Shen et al., 2013). Las configuraciones más utilizadas tienen únicamente excitación mediante imanes permanentes. En la Figura 6.b se muestra una máquina trifásica 12/10, esto es con 12 polos en el estator y 10 dientes en el rotor. Cada polo del estator consta de un devanado concentrado y un imán permanente que está magnetizado en la dirección tangencial. Los imanes permanentes en los polos adyacentes están magnetizados en direcciones opuestas con el fin de obtener concentración de flujo en el entrehierro (Shi et al., 2016).

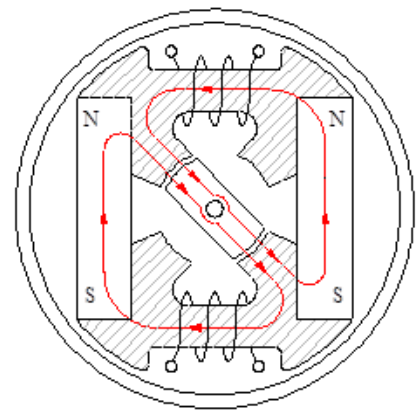

a)

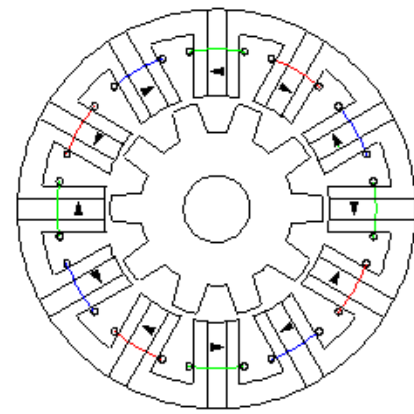

b)

Fig. 6: a) Configuración original del alternador con conmutación de flujo; b) Configuración trifásica, 12/10, del alternador con conmutación de flujo excitado mediante imanes permanentes.

En la Figura 7 se muestra el principio de funcionamiento de los ACF, en los que, cuando el rotor se mueve, las líneas de flujo son guiadas alternativamente a ambos lados de los imanes permanentes. En la Figura 7.a. un diente del rotor se alinea con uno de los dos dientes del estator sobre los cuales se encuentra enrollada una bobina del inducido. En la figura 7.b. el rotor se mueve hacia la derecha, alineándose un diente del rotor con el otro diente del estator correspondiente a la misma bobina. En ambos casos, el flujo ligado a la bobina tiene el mismo valor, pero su polaridad se ha invertido. La frecuencia de la tensión inducida viene dada, como en los Al, por $f=Z_{r} \cdot n / 60$, siendo $Z_{r}$ el número de dientes del rotor y $n$ la velocidad de rotación, en rpm.

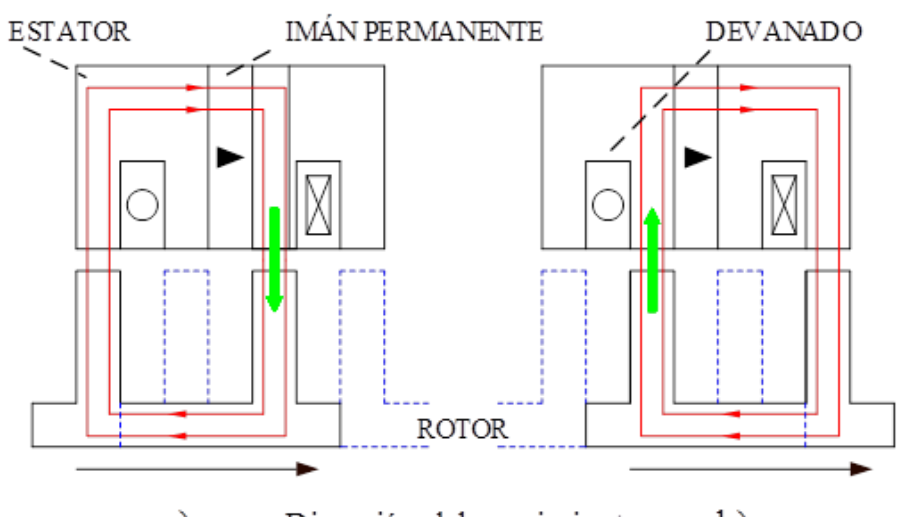

a) Dirección del movimiento

b)

Fig. 7: Principio de conmutación del flujo.

Cuando se utiliza la máquina como generador de alta velocidad, resulta difícil regular el valor del campo magnético producido por los imanes ( $\mathrm{Li}$ et al., 2016). Esto hace que, en algunas aplicaciones, sea aconsejable utilizar un devanado de corriente continua para la excitación, en lugar de imanes permanentes (Zhu et al., 2015). La excitación híbrida combina las ventajas del devanado de corriente continua y de los imanes permanentes y permite mejor control del campo magnético (Gausens et al., 2014). Las principales ventajas del ACF, en aplicaciones de alta velocidad, son: Alta densidad de potencia, alta eficiencia, estructura compacta y robusta como consecuencia de tener los imanes en el estator, simplicidad del rotor, funcionamiento en un amplio rango de velocidades, alta capacidad de tolerancia ante fallos, onda sinusoidal de la tensión inducida y facilidad de enfriamiento. El principal inconveniente es que, al igual que ocurre en los $\mathrm{Al}$, la frecuencia de salida para una misma velocidad de rotación es mayor que la que se obtendría con un generador síncrono convencional. Otras desventajas están relacionadas con la utilización de imanes permanentes como sistema de excitación, (coste y desmagnetización), y con los problemas inherentes al sistema de control, a las que hay que añadir, en los sistemas híbridos, la complejidad de su diseño. 


\section{EVALUACIÓN CRÍTICA DE LOS GENERADORES SINCRONOS DE ALTA VELOCIDAD}

La elección de un generador de alta velocidad, para una aplicación determinada, está fuertemente condicionada por los requerimientos específicos, (potencia de salida y rango de velocidades de operación), y los particulares de cada aplicación. Así por ejemplo, en el caso de los generadores para aviación, son fundamentales la fiabilidad y la densidad de potencia, mientras que en el guiado de misiles, con un funcionamiento durante un breve espacio de tiempo que acaba con su destrucción final, lo son la simplicidad y el coste económico. Entre los tipos considerados: ASIP, AlHO y ACF se ha realizado una evaluación crítica que derivará en una comparativa entre ellos acerca de su mayor o menor idoneidad para las aplicaciones que requieren alta velocidad de rotación. (Los AlHE se consideran configuraciones particulares de ACF).

Para ello se han seleccionado una serie de requisitos, en base a lo expuesto anteriormente, que se considera que han de verificar los generadores síncronos de alta velocidad. Estos requisitos se han clasificado en cinco grupos distintos. El primer grupo agrupa a los requisitos específicos de diseño, (simplicidad constructiva, desmagnetización de los imanes, reversibilidad, capacidad de tolerancia ante fallos, coste y fiabilidad). El segundo grupo engloba a los parámetros de dimensionamiento, (máxima velocidad periférica, densidad de potencia, carga magnética específica y carga eléctrica específica). El tercer grupo incluye los requisitos relativos a las pérdidas y a la refrigeración, (pérdidas eléctricas y magnéticas del estator y del rotor, pérdidas mecánicas, eficiencia y facilidad de refrigeración). El cuarto grupo a los requisitos relacionados con la electrónica de potencia y el control auxiliar, (armónicos en la tensión, facilidad de regulación de la tensión y simplicidad del convertidor electrónico de potencia y de la electrónica auxiliar). Finalmente el quinto grupo se refiere a los requisitos medioambientales, (capacidad para trabajar en ambientes adversos, ruido y vibración).

Se han valorado por igual todos los requisitos (10). La puntuación asignada es más alta cuanto más idóneo se considera al generador en cuestión, para el requisito indicado. El análisis de los aspectos constructivos y de funcionamiento de las diferentes máquinas, permite establecer una comparación relativa entre ellas, y determinar el grado de satisfacción para cada requisito. Se ha realizado, además, una selección de la información que puede extraerse revisando la bibliografía. Al aplicar valores numéricos se obtiene una valoración global que cuantifica, de forma relativa, la idoneidad, para el funcionamiento a alta velocidad de rotación, de los tipos de generadores que son objeto de estudio. En la Tabla 3 se muestran los resultados de la comparación.

Tabla 3: Comparación de los generadores síncronos de alta velocidad.

\begin{tabular}{|l|c|c|c|}
\hline \multicolumn{1}{|c|}{ Requisito } & ASIP & AlHO & ACF \\
\hline Simplicidad constructiva & 7 & 6 & 8 \\
Desmagnetización de los imanes & 7 & 10 & 8 \\
Reversibilidad & 9 & 7 & 7 \\
Capacidad de tolerancia ante fallos & 8 & 9 & 9 \\
Coste & 6 & 7 & 7 \\
Fiabilidad & 8 & 9 & 8 \\
Máxima velocidad periférica & 7 & 9 & 9 \\
Densidad de potencia & 10 & 6 & 7 \\
Carga magnética espeć́fica & 9 & 6 & 8 \\
Carga eléctrica específica & 8 & 7 & 7 \\
Pérdidas eléctricas y magnéticas del estator & 8 & 7 & 8 \\
Pérdidas eléctricas y magnéticas del rotor & 8 & 9 & 8 \\
Pérdidas mecánicas & 9 & 7 & 7 \\
Eficiencia & 10 & 6 & 8 \\
Facilidad de refrigeración & 7 & 9 & 9 \\
Armónicos en la tensión & 8 & 7 & 8 \\
Facilidad de regulación de la tensión & 7 & 9 & 7 \\
Simplicidad del convertidor electrónico de potencia & 7 & 7 & 7 \\
Capacidad para trabajar en ambientes adversos & 7 & 9 & 8 \\
Ruido y vibración & 10 & 8 & 7 \\
\hline Valoración Global & 160 & 154 & 155 \\
\hline
\end{tabular}


Como se desprende de las puntuaciones totales alcanzadas en cada caso, los ASIP constituyen la mejor opción, sobre todo por su eficiencia, densidad de potencia y pérdidas mecánicas y también, como se deduce de los resultados recogidos en la Figura 2, por su idoneidad para trabajar en todo el rango de altas velocidades. Además, su configuración bipolar permite generar, para una misma velocidad de rotación, una tensión con menor frecuencia que la de cualquier otro tipo de generador síncrono. Los principales inconvenientes de los ASIP radican en la disposición y fijación en el rotor de los imanes permanentes y en el riesgo de desimantación por los efectos desmagnetizantes de las elevadas corrientes del inducido y/o las altas temperaturas. Sin embargo, en el margen bajo-medio de las altas velocidades de rotación, (10.000 $50.000 \mathrm{rpm}$ ), determinados requisitos específicos pueden equilibrar las desventajas de los AlHO o ACF con respecto a los ASIP convirtiéndolos en la mejor opción.

\section{CONCLUSIONES}

En este artículo, tras exponer los aspectos que condicionan el diseño de las máquinas eléctricas de alta velocidad, se describen los diferentes generadores síncronos para aplicaciones aeroespaciales, de almacenamiento de energía y de generación con microturbinas y se realiza una evaluación critica de los generadores síncronos más idóneos para la alta velocidad de rotación. Aunque la decisión de la elección de un generador está muy condicionada por la aplicación considerada, puede afirmarse que los generadores síncronos con imanes permanentes en el rotor son la mejor opción, especialmente para el caso de las velocidades más elevadas. Sin embargo, los generadores con conmutación de flujo y los alternadores inductores homopolares pueden ser una buena elección, para algunas aplicaciones, en el rango bajo-medio de las altas velocidades de rotación, (10.000 - 50.000 rpm).

\section{REFERENCIAS}

Antipov, V. N. y Danilevich, Y. B., High-speed electrical machines for power engineering: current state and development trends, doi: 10.3103/S1068371207060016, Russian Electrical Engineering, 78 (6), 277 - 279 (2007)

Arumugan, P., Dusek, J. y otros seis autores, Comparative design analysis of permanent magnet rotor topologies for an aircraft starter-generator, doi: 10.1109/IEPS.2014.6874194, IEEE International Conference on Intelligent Energy and Power Systems, 273 - 278, Kiev (Ucrania), 2 al 6 de Junio (2014)

Bartolo, J. B., Zhang, H. y otros tres autores, High speed electrical generators, application, materials and design, doi: 10.1109/WEMDCD.2013.6525164, IEEE Workshop on Electrical Machines Design Control and Diagnosis, 47 - 59, París (Francia), 11 al 12 de Marzo (2013)

Binder, A. y Canders, W., High speed drives, ISBN: 978-1-4799-4389-0, International Conference on Electrical Machines, Tutorial, Berlín (Alemania), 2 al 5 de Septiembre (2014)

Binder, A. y Schneider, T., High-speed inverter-fed AC drives, doi: 10.1109/ACEMP.2007.4510476, International Aegean Conference on Electrical Machines and Power Electronics, 9 - 16, Bodrum (Turquía), 10 al 12 de Septiembre (2007)

Binder, A., Schneider, T. y Klohr, M., Fixation of buried and surface mounted magnets in high speed permanent magnet synchronous machines, doi: 10.1109/TIA.2006.876072, IEEE Trans on Industry Applications, 42 (4), 1031 - 1037 (2006)

Borisavljevic, A., Limits, modeling and design of high-speed permanent magnet machines, ISBN: 978-908570-837-7, Ph. D. Tesis, Technische Universiteit Delft, Países Bajos (2011)

Davies, E. J. y Higginson, A. M., Airgap and stator flux distribution in Guy-type medium frequency inductor generators, doi: 10.1049/piee.1975.0105, Proceedings of the Institution of Electrical Engineers, 122 (4), 377 - 386 (1975)

Davies, E. J. y Lay, R. K., Performance of Lorentz-type medium frequency inductor alternators on load, doi: 10.1049/piee.1968.0313, Proceedings of the Institution of Electrical Engineers, 115 (12), 1791 - 1800 (1968)

Ede, J. D., Zhu, Z. Q. y Howe, D., Rotor resonances of high-speed permanent magnet brushless machines, doi: 10.1109/TIA.2002.804765, IEEE Trans on Industry Applications, 38 (6), 1542 - 1548 (2002)

Fernando, N., Vakil, G. y otros cuatro autores, Impact of soft magnetic material on design of high speed permanent magnet machines, doi: 10.1109/TIE.2016.2587815, IEEE Trans on Industrial Electronics, ISSN 1557-9948 (2016) 
Ganev, E., Selecting the best electric machines for electrical power-generation systems: High-performance solutions for aerospace more electric architectures, doi: 10.1109/MELE.2014.2364731, IEEE Electrification Magazine, 2 (4), 13 - 22 (2014)

Gaussens, B., Hoang, E. y otros tres autores, A hybrid-excited flux-switching machine for high-speed DCalternator applications, doi: 10.1109/TIE.2013.2281152, IEEE Trans on Industrial Electronics, 61 (6), 2976 2989 (2014)

Gerada, D., Mebarki, A. y otros cuatro autores, High-speed electrical machines: Technologies, trends and developments, doi: 10.1109/TIE.2013.2286777, IEEE Trans on Industrial Electronics, 61 (6), 2946 - 2959 (2014)

Gieras, J. F., Design of permanent magnet brushless motors for high speed applications, doi: 10.1109/ICEMS.2014.7013920, 17 th International Conference on Electrical Machines and Systems, 1 - 16, Hangzhou (China), 22 al 25 de Octubre (2014)

Gieras, J. F., New applications of synchronous generators, ISSN: 0033-2097, Przeglad Elektrotechniczny (Electrical Review), 88 (9), 150 - 157 (2012)

Huang, Z. y Fang, J., Multiphysics design and optimization of high-speed permanent-magnet electrical machines for air blower applications, doi: 10.1109/TIE.2016.2518121, IEEE Trans on Industrial Electronics, 63 (5), 2766 - 2774 (2016)

Huynh, C., Zheng, L. y Acharya, D., Losses in high speed permanent magnet machines used in microturbine applications, doi:10.1115/1.2982151, Journal of Engineering for Gas Turbines and Power, 131 (2), Art. № 022301 (2009)

Kim, K. H., Park, H. I. y otros tres autores, Comparative study of electromagnetic performance of high-speed synchronous motors with rare-earth and ferrite permanent magnets, doi: 10.1109/TMAG.2016.2532901, IEEE Trans on Magnetics, 52 (7), Art. № 8203404 (2016)

Kumar, S., Lipo, T. A. y Kwon, B., A 32,000 rev/min axial flux permanent magnet machine for energy storage with mechanical stress analysis, doi: 10.1109/TMAG.2015.2512939, IEEE Trans on Magnetics, 52 (7), Art № 8205004 (2016)

Lahne, H. C., Gerling, D. y otros dos autores, Design of a 50000 rpm high-speed high-power six-phase PMSM for use in aircraft applications, doi: 10.1109/EVER.2016.7476345, $11^{\text {th }}$ International Conference on Ecological Vehicles and Renewable Energies, 1 - 11, Montecarlo (Monaco), 6 al 8 de Abril (2016)

Leão, A., Generador eólico superconductor, doi: 10.1612/inf.tecnol.4111it.08, Información Tecnológica, 21 (2), 37 - 44 (2010)

Li, S., Li, Y. y otros dos autores, High speed electric machines. Challenges and design considerations, doi: 10.1109/TTE.2016.2523879, IEEE Trans on Transportation Electrification, 2 (1), 2 - 13 (2016)

Li, Y., Bobba, D. y Sarlioglu, B., A novel 6/4 flux-switching permanent magnet machine designed for highspeed operations, doi: 10.1109/TMAG.2016.2547366, IEEE Trans on Magnetics, 52 (8), Art No 8107109 (2016)

Maeda, A., Tomita, H. y Miyashita, O., Power and speed limitations in high speed electrical machines, http://library.mit.edu/item/001352668, International Power Electronics Conference, 1321 - 1326, Yokohama (Japón), 3 al 7 de Abril (1995)

McNab, I. R., Pulsed power options for large EM launchers, doi: 10.1109/TPS.2014.2372173, IEEE Trans on Plasma Science, 43 (5), 1352 - 1357 (2015)

Milspec Manufacturing Pty Ltd, Albury (Australia), en la web: http://milspecmanufacturing.com/products/ alternators/360-ac/. Último acceso: 3 Agosto (2016)

Moghaddam, R. R., High speed operation of electrical machines, a review on technology, benefits and challenges, doi: 10.1109/ECCE.2014.6954160, IEEE Energy Conversion Congress and Exposition, 5539 5546, Pittsburgh (USA), 14 al 18 de Septiembre (2014)

Pillai, K. P., Nair, A. S. y Bindu, G. R., Unbalanced magnetic pull in train-lighting brushless alternators with static eccentricity, doi: 10.1109/TVT.2007.901966, IEEE Trans on Vehicular Technology, 57 (1), 120 - 126 (2008) 
Rahman, M. A., Chiba, A. y Fukao, T., Super high speed electrical machines - Summary, doi: 10.1109/PES.2004.1373062, IEEE Power Engineering Society General Meeting, 1272 - 1275, Denver (USA), 6 al 10 de Junio (2004)

Rauch, S. E. y Johnson, L. J., Design principles of flux switch alternators, doi: 10.1109/ AIEEPAS.1955.4499226, IEE Trans Power Apparatus and Systems, Part III, 74 (3), 1261 - 1268 (1955)

Reddy, P. B., Jahns, T. M. y Bohn, T. P., Transposition effects on bundle proximity losses in high-speed PM machines, doi: 10.1109/ECCE.2009.5316037, IEEE Energy Conversion Congress and Exposition, 1919 1926, San José (USA), 20 al 24 de Septiembre (2009)

Severson, E., Nilssen, R. y otros dos autores, Magnetic equivalent circuit modeling of AC homopolar machine for flywheel energy storage, doi: 10.1109/TEC.2015.2441040, IEEE Trans on Energy Conversion, 30 (4), 1670 - 1678 (2015)

Shen, J. X. y Fei, W. Z., Permanent magnet flux switching machines. Topologies, analysis and optimization, doi: 10.1109/PowerEng.2013.6635633, th $^{\text {th }}$ International Conference on Power Engineering, Energy and Electrical Drives, 352 - 366, Estambul (Turquía), 13 al 17 de Mayo (2013)

Shi, Y., Jian, L. y otros cuatro autores, A new perspective on the operating principle of flux-switching permanent-magnet machines, doi: 10.1109/TIE.2015.2492940, IEEE Trans on Industrial Electronics, 63 (3), 1425 - $1437(2016)$

Sivasubramaniam, K., Zhang, T. y otros seis autores, Development of a high speed HTS generator for airborne applications, doi: 10.1109/TASC.2009.2017758, IEEE Trans on Applied Superconductivity, 19 (3), 1656 - 1661 (2009)

Tenconi, A., Vaschetto, S. y Vigliani, A., Electrical machines for high-speed applications: Design considerations and tradeoffs, doi: 10.1109/TIE.2013.2276769, IEEE Trans on Industrial Electronics, 61 (6), 3022 - 3029 (2014)

Tüysüz, A., Steichen, M. y otros dos autores, Advanced cooling concepts for ultra-high speed machines, doi: 10.1109/ICPE.2015.7168081, $9^{\text {th }}$ International Conference on Power Electronics, 2194 - 2202, Seul (Korea), 1 al 5 de Junio (2015)

Uzhegov, N., Nerg, J. y otros cuatro autores, Multidisciplinary design process of a 6-slot 2-pole high-speed permanent-magnet synchronous machine, doi: 10.1109/TIE.2015.2477797, IEEE Trans on Industrial Electronics, 63 (2), 784 - 795 (2016)

Walker, J. H., High frequency alternators, doi: 10.1049/ji-2.1946.0016, Journal of the Institution of Electrical Engineers. Part II: Power Engineering, 93 (31), 67 - 80 (1946)

Walker, J. H., The theory of the inductor alternator, doi: 10.1049/ji-2.1942.0037, Journal of the Institution of Electrical Engineers, 89 (9), 227 - 241 (1942)

Wrobel, R., Vainel, G. y otros cuatro autores, Investigation on mechanical loss components and heat transfer in an axial-flux PM machine, doi: 10.1109/TIA.2015.2405499, IEEE Trans on Industry Applications, 51 (4), $3000-3011(2015)$

Ye, C., Yu, K. y otros dos autores, Optimal design and experimental research of a capacitor-charging pulsed alternator, doi: 10.1109/TEC.2015.2395446, IEEE Trans on Energy Conversion, 30 (3), 948 - 956 (2015)

Zhang, F., Du, G. y otros cuatro autores, Electromagnetic design and loss calculations of a $1.12 \mathrm{MW}$ highspeed permanent-magnet motor for compressor applications, doi: 10.1109/TEC.2015.2488841, IEEE Trans on Energy Conversion, 31 (1), 132 - 140 (2016)

Zhang, F., Du, G. y otros tres autores, Rotor retaining sleeve design for a $1.12 \mathrm{MW}$ high-speed PM machine, doi: 10.1109/TIA.2015.2423659, IEEE Trans on Industry Applications, 51 (5), 3675 - 3685 (2015)

Zheng, H., High frequency AC power systems, en la web: http://scholarcommons.sc.edu/etd/2684, Ph. D. Tesis, University of South Carolina, USA (2014)

Zhu, Z. Q., Zhou, Y. J. y otros dos autores, Investigation of nonoverlapping stator wound-field synchronous machines, doi: 10.1109/TEC.2015.2423280, IEEE Trans on Energy Conversion, 30 (4), 1420 - 1427 (2015)

Zwyssig, C., Kolar, J. W. y Round, S. D., Mega-speed drive systems: Pushing beyond 1 million r/min, doi: 10.1109/TMECH.2008.2009310, IEEE/ASME Trans on Mechatronics, 14 (5), 564 - 574 (2009) 